\title{
30 YEARS OF THE MINERALOCORTICOID RECEPTOR Mineralocorticoid receptor activation and specificity-conferring mechanisms: a brief history
}

\author{
John W Funder \\ Hudson Institute, Monash Medical Centre, and Monash University, Clayton, Australia
}

Correspondence

should be addressed

to J Funder

Email

john.funder@hudson.org.au
The mineralocorticoid receptor (MR) was first characterized 45 years ago (Funder 1972, Rousseau 1972), a decade and a half before its successful cloning (Arriza 1987) to which this issue of the Journal is dedicated. Aldosterone had been isolated and characterized 20 years before the MR: its initial name was electrocortin, reflecting its ability to promote electrolyte transport across epithelial membranes, rapidly changed to its familiar, current name aldosterone. What prompted the change was the finding of a unique aldehyde group at carbon 18 of the steroid molecule, the physiological significance of which only became clear many years later (Edwards 1988, Funder 1988).

Not surprisingly, given the rapidly established role of aldosterone in the transepithelial transport of sodium, potassium and water, was that the early running was made by nephrologists and renal physiologists. Although rapid effects of aldosterone on renal sodium transport were reported in early studies (Ganong 1958), later attention focused on the demonstration that aldosterone action could be blocked at the level of DNA transcription (by actinomycin D) or mRNA translation (by puromycin), evidence that aldosterone acted principally at the intracellular level by DNA-directed, RNA-mediated protein synthesis.

This expanded the field of interest beyond the nephrologists to include cell biologists, endocrinologists and clinicians - evidence for the latter are the initial reports of dexamethasone suppressible hyperaldosteronism (Sutherland 1966, New 1967). These were published years before the receptor was characterized by high affinity, displaceable (sometimes bravely termed specific) binding of tritiated aldosterone in kidney slices from adrenalectomized rats; less convincing, in retrospect, was a similar report in rat kidney cytosol (Rousseau 1972), given the subsequent demonstration that molybdate is needed for cytosolic MR to survive in vitro (Emadian 1988).

The interest of endocrinologists was kindled by the recognition that the MR was one of a family of nuclear transactiving factors, which includes glucocorticoid receptors (GR), progesterone receptors (PR), androgen receptors, and more distantly estrogen receptors and those for 1,25 dihydroxycholecalciferol. Interest was further kindled in the case of the MR by the demonstration of its presence in tissues not primarily involved in transepithelial transport - e.g., brain, heart and blood vessels (Krozowski 1981, Pearce 1987, Funder 1989).

At the same time a series of studies showed that MR had not only (high picomolar) affinity for aldosterone, but also puzzlingly equally high affinity for cortisol, corticosterone, deoxycorticosterone and progesterone. Such studies needed to be carefully designed: transcortin in fetal calf serum lowers values for cortisol and corticosterone, and studies on progesterone binding require siliconized syringes and glassware. Neither of these latter are available in vivo, but the potent MR antagonist effect of elevated progesterone in pregnancy, to raise countervailing aldosterone levels 3-10 fold, had been noted very early and provided the point of departure for the synthesis of spironolactone (Kagawa 1957). Similarly, deoxycorticosterone had been recognized as the 'standard' mineralocorticoid hormone more than a decade before it was supplanted by the physiologic mineralocorticoid aldosterone.

The implications of this MR promiscuity were immediately clear. In high blood flow organs, such as

This paper is part of a thematic review section on 30 Years of the Mineralocorticoid Receptor. The guest editors for this section were

John Funder and Maria,Ghristina Zemnargioscientifica.com at 04/26/2023 06:21:34AM 
the kidney, intracellular levels of hormones derive from what circulates free in the plasma: differential binding to plasma protein - transcortin (CBG) and albumin - was recognized early on as a factor. Aldosterone and deoxycorticosterone (DOC) circulate at more or less similar levels, although primarily in response to different secretagogues. Aldosterone is $\sim 50 \%$ bound to albumin in plasma, DOC is $98-99 \%$ bound: in vivo studies show DOC to be one fortieth as potent a mineralocorticoid as aldosterone. The effects of progesterone in pregnancy have been cited: lesser effects in the post-ovulatory phase of the menstrual cycle may produce a false positive level of aldosterone mimicking primary aldosteronism (Ahmed 2011).

The problem is with the physiologic glucocorticoids. Plasma binding goes some way - but certainly not all the way - in lowering their free levels. Total circulating physiologic glucocorticoid levels are $\sim 1000$-fold higher than those of aldosterone on a normal diet: in contrast with aldosterone, rather than being $~ 50 \%$ plasma bound, they are $\sim 95 \%$ : free plasma levels are thus 'only' $\sim 100$ times those of aldosterone. We know that aldosterone regulates sodium and water homeostasis via MR in the distal tubular/collecting duct principal cells: the question is how can it access the MR in the face of a 100-fold higher concentration of glucocorticoids?

Two independent studies delineated the mechanism, though both (Edwards 1988, Funder 1988) in a sense got it only half right at the time. The enzyme 11 $\beta$-hydroxysteroid dehydrogenase (henceforth 11ßHSD2) is co-expressed at high levels in principal cells (and other classical aldosterone target tissues). The enzyme acts, essentially unidirectionally, to convert cortisol to cortisone and corticosterone to 11-keto corticosterone: neither product has appreciable affinity for MR. Aldosterone is not a substrate for $11 \beta \mathrm{HSD} 2$, in that whereas it can be drawn on paper with a $\mathrm{CHO}$ rather than the usual (for 21 carbon steroids) methyl group at $\mathrm{C} 18$, in plasma the aldehyde group cyclizes with the hydroxyl on carbon 11, forming an 11,18 hemiacetal or $11,18,20$ hemiketal, which accounts for $\sim 99 \%$ of circulating aldosterone. It is this unique $\mathrm{CHO}$ at $\mathrm{C} 18$ which allows aldosterone to bypass metabolism by $11 \beta \mathrm{HSD} 2$ and thus activate MR in the principal cells of the nephron.

So far, so good: if the $11 \beta \mathrm{HSD} 2$ is deficient, as in pseudohyperaldosteronism, or blocked by glycyrrhizic/ glycyrrhetinic acid in liquorice over-consumption, cortisol activates the 'unprotected' tubular MR. Where both initial studies proven in retrospect to be deficient was that they presumed that 11ßHSD2 metabolism of cortisol/ corticosterone blocked MR occupancy by glucocorticoids, given its high level of co-expression and high affinity for glucocorticoids. While this may be satisfyingly simple, mature reflection prompted a re-examination of the selective ability of aldosterone to activate protected MR under normal conditions, and that of cortisol to mimic aldosterone when $11 \beta \mathrm{HSD} 2$ is blocked or deficient.

First is simple mathematics. If cortisol is normally an agonist that must be excluded from renal MR let us say that a possibly acceptable level of 'noise' in the MR response would be were cortisol levels to be reduced to $~ 10 \%$ those of aldosterone. What this would require is for 999 of every 1000 incident cortisol molecules to be converted to cortisone, in an organ which receives $20-25 \%$ of the cardiac output. In adrenalectomized rats injected with ${ }^{3} \mathrm{H}$-aldosterone alone, or plus progressively increasing doses of non-radioactive aldosterone or corticosterone, the latter proved to be an order of magnitude less potent that aldosterone in competing for tracer binding, consistent with its 10-fold higher plasma binding (Funder \& Myles 1996). What this translates to in vivo is that $11 \beta \mathrm{HSD} 2$ debulks intracellular glucocorticoid levels, from 100fold those of aldosterone to 10 -fold: a corollary of this is that under normal conditions $\sim 90 \%$ of 'protected' MR are occupied but not activated by glucocorticoid. Consistent with this is the Kagawa assay for MR antagonist action: adrenalectomy vs salt loading shifts the dose-response curve an order of magnitude to the left, reflecting full MR 'availability.'

How can it be that cortisol is not a renal MR agonist under normal conditions, despite occupying $~ 90 \%$ of the receptors, but becomes an MR when 11ßHSD2 activity is deficient? Ockham's razor would suggest that the simplest answer might lie in the commonly overlooked obligate cosubstrate for $11 \beta \mathrm{HSD} 2$, nicotinamide adenine dinucleotide (NAD). For every molecule of cortisol converted to cortisone, one molecule of NAD is converted to NADH. NAD levels vary between cells and intracellular compartments, but under normal circumstances a ratio of 600:1 for NAD:NADH is a reasonable estimate. On this basis, a very active enzyme converting 9 of every 10 incident cortisol molecules to cortisone might raise NADH levels 100 -fold, with only a modest change in substrate NAD. There is evidence for an increase in NADH levels blocking activity of other nuclear transactivating factors (Fjeld et al. 2003); evidence that similar changes in redox state are operant for cortisol is indirect but compelling.

There is clear evidence that there are between-cell and between-tissue differences in the MR response to aldosterone and cortisol, as reviewed in detail in this

Published by Bioscientifica Ltd 
commemorative issue (Fuller 2017). These differences - in post-binding structural conformation of the ligandbinding domain, $\mathrm{N}-\mathrm{C}$ terminal interaction, co-regulator recruitment - presumably are responsible for the betweenligand differentiation of effects in terms of physiology; in terms of pathophysiology we are dealing with the same tissue, the same cell, but differences in the cellular milieu. This has been clearly shown by the use of the widely used model, the Langendorf rat heart ischemiareperfusion preparation. The circumflex or left anterior descending coronary artery is ligated for $30 \mathrm{~min}$, followed by reperfusion for two hours: administered steroids are given $15 \mathrm{~min}$ before ligation. At the end of the reperfusion the hearts are taken and area-at-risk and infarct size are determined (Mihailidou et al. 2009).

Aldosterone increased both infarct size and area-at-risk above ischemia alone, changes reversed by the antioxidant tempol and spironolactone, as previously reported from the Danser laboratory (Chai et al. 2005). What is novel about this series of studies are two things. First, low-dose cortisol mimics the deleterious effect of aldosterone in the context of tissue damage: like aldosterone, the cortisol effect is reversed by spironolactone, but not by the GR, PR antagonist RU38486. Second is something unexpected for spironolactone, not particularly relevant to the cortisol:aldosterone paradox, but crucial in interpreting the RALES trial in heart failure.

In the standard Langendorf preparation spironolactone alone, without any other steroid, reduces area-at-risk and infarct size (Mihailidou et al. 2009). In rats a week after adrenalectomy, to exclude the possible but improbable effects of retained endogenous steroids, the animals are more fragile, but again spironolactone significantly lowers infarct size and area-at-risk. The interpretation of these findings is that rather than a 'blocker', denying aldosterone (or cortisol) access to MR, spironolactone is a so-called inverse agonist, whereby it produces its effects at surprisingly low concentrations.

Where this is particularly relevant is in considering the mechanisms underlying the prismatic RALES trial (Pitt et al. 1999). In this trial low-dose spironolactone (mean dose $26 \mathrm{mg} /$ day) was added to standard of care for patients with progressive, largely NYHA Class 3 , congestive heart failure. After 18 months the trial was terminated, as the spironolactone group showed 30\% lower mortality than the control group, and 35\% fewer hospitalizations. This was hailed, and is still widely cited, as evidence of spironolactone being able to exclude aldosterone from myocardial MR; there are several facets of the trial that make this conclusion unsupportable. First, despite a proportion of the patients being on diuretics, plasma aldosterone concentrations were in the low normal range. Secondly, and crucially, cardiomyocytes do not express $11 \beta \mathrm{HSD} 2$, so that their MR are overwhelmingly occupied by cortisol, and activated by cortisol in the context of tissue damage.

It is commonly assumed that spironolactone as an antagonist in epithelia acts as a blocker, denying aldosterone access to MR. The affinity of spironolactone in vitro is one fourth that of aldosterone, and steady state plasma concentrations of spironolactone (which in vivo is short-lived) together with its principal metabolites, of which canrenone has the longest half-life, are $\sim 100 \mathrm{nM}$ after a $25 \mathrm{mg}$ oral dose. At first blush this would appear to support a simple 'blocking' mechanism given the nM concentrations of aldosterone - except that spironolactone is $~ 90 \%$ plasma bound, and canrenone $>99 \%$ plasma bound. Blockade may still be a major determinant of antagonist activity in aldosterone target tissues, despite the lower affinity and much higher plasma binding of the antagonists: it is also possible that the inverse agonist action of spironolactone also plays a part.

With cortisol as the MR agonist in the context of tissue damage, it is not possible that the free levels of spironolactone and its metabolites can occupy more than $10-15 \%$ of the cardiac MR, given the relative affinities and extent of plasma binding: it cannot have its effect primarily by denying cortisol access. What spironolactone did in the RALES trial was to occupy some of the cardiomyocyte $\mathrm{MR}$, and - acting again as an inverse agonist - mitigate the tissue damage-induced MR agonist action of cortisol: aldosterone is not even in the picture. What this means is that what holds in the rat whole heart Langendorff model also holds in vivo in the failing human heart, with its ROS generation and consequent redox change in response to tissue damage.

Where does this take us? The answer is a radical reconsideration of the physiology and pathophysiology of MR and their ligands, agonist and antagonist. The time-honored physiology of aldosterone acting at epithelia to regulate fluid and electrolyte balance remains intact: everything else is essentially up for grabs. The first evidence that MR is not unique receptor for aldosterone is their appearance, in cartilaginous and bony fish, millions of years before that of aldosterone, a terrestrial hormone first found in lungfish (Kassahn et al. 2011). As their name suggests such creatures have both gills and lungs, and represent the emergence from an obligate aqueous
Published by Bioscientifica Ltd 
environment to facultative air breathing. In trout and tilapia and zebra fish the physiologic role(s) of MR are still unclear: the one thing we can be sure of is that they are not receptors for aldosterone. We ignore the lessons of evolution at our peril.

Given their widespread distribution in nonepithelial tissue, and their overwhelming occupancy by glucocorticoids, it is obviously incorrect to term MR generically as 'aldosterone receptors'. The initial term of 'type 1 corticosteroid receptor' is both clunky and beyond resuscitation: it has been replaced by the currently accepted nomenclature of 'mineralocorticoid receptor'. Even in the kidney this is a better term than 'aldosterone receptor', in that cortisol acts as a mineralocorticoid in principal cells when $11 \beta \mathrm{HSD} 2$ is deficient or in intercalated cells when MR phosphorylated at $S^{843}$ are dephosphorylated in response to high levels of plasma angiotensin (Shibata et al. 2013).

Much of the cell and molecular biology of aldosterone and of the MR has been established in vitro, and the physiology and pathophysiology in adrenalectomized animals. Interpretation of these studies is always limited, and often compromised, by the absence of the physiologic glucocorticoids at free concentrations $~ 100$-fold those of aldosterone. Variation on this theme are the use of industrial doses of aldosterone (e.g. 10x the elevated secretion of sodium deficient rats) plus $4-8 \%$ sodium chloride to drink in studies on adrenal-intact animals, with carefree extrapolation of the resulting findings as relevant to the cardiovascular pathophysiology and even physiology of aldosterone and MR.

Finally, there are clinical implications aplenty. If cortisol acts as an MR 'agonist' in damaged tissues, there may logically be a place for spironolactone (or its later congeners) in conditions in addition to its proven clinical roles. Spironolactone is a dirty drug, with anti-androgen and progestational side-effects making it sub-optimal for widespread use in other than primary aldosteronism resistant hypertension and progressive cardiac failure. Clinical trials of more specific MR antagonists would thus be welcome as potentially (and preferably) modestly priced alternatives to current antibody-based antiinflammatory drugs. A corollary of the pro-inflammatory effect of cortisol in tissue damage is a hitherto probably under-recognized double anti-inflammatory effect of potent synthetic GR-selective glucocorticoids. Dexamethasone, for example, may act not only via GR as an anti-inflammatory, but also by supressing the secretion of endogenous cortisol to levels that may possibly result in lower MR activation.
In conclusion, then, central to any consideration of specificity-conferring mechanisms for MR is its bivalent relationship with cortisol, its almost certain primordial ligand. We are used to considering aldosterone as the touchstone mineralocorticoid: what we need to fathom is not only how NADH generation stills cortisol-MR complexes in the normal kidney, but also to compare the expression signatures of cortisol as an MR antagonist in the normal cardiomyocyte with those of spironolactone. Thirty years ago Ron Evans and coworkers succeeded in cloning the MR by techniques laborious by today's standards. To probe how cortisol is a context-dependent bivalent MR ligand, and to seek physiologic and pathophysiologic insight from their findings, should be well within the competence to the molecular biologists (and pathophysiologists) of 2017.

\section{Declaration of interest}

The author declares that there is no conflict of interest that could be perceived as prejudicing the impartiality of this review.

\section{Funding}

This research was supported in part by the Victorian Government's Victorian Infrastructure Fund.

\section{References}

Ahmed AH, Gordon RD, Taylor PJ, Ward G, Pimenta E \& Stowasser MJ 2011 Are women more at risk of false-positive primary aldosteronism screening and unnecessary suppression testing than men? Journal of Clinical Endocrinology and Metabolism 96 E340-E346. (doi:10.1210/ jc.2010-1355, Epub 2010 Oct 20)

Arriza JL, Weinberger C, Cerelli G, Glaser TM, Handelin BL, Housman DE \& Evans RM 1987 Cloning of human mineralocorticoid receptor complementary DNA: structural and functional kinship with the glucocorticoid receptor. Science 237 268-275. (doi:10.1126/ science.3037703)

Chai W, Garrelds IM, Arulmani U, Schoemaker RG, Lamers JM \& Danser AH 2005 Genomic and nongenomic effects of aldosterone in the rat heart: why is spironolactone cardioprotective? British Journal of Pharmacology 145 664-671. (doi:10.1038/sj.bjp.0706220)

Edwards CR, Stewart PM, Burt D, Brett L, McIntyre MA, Sutanto WS, de Kloet ER \& Monder C 1988 Localisation of 11 beta-hydroxysteroid dehydrogenase - tissue specific protector of the mineralocorticoid receptor. Lancet 2 986-989. (doi:10.1016/S0140-6736(88)90742-8)

Emadian SM \& Luttge WG 1988 A novel effect of molybdate on the binding of $[3 \mathrm{H}]$ aldosterone to gel-filtered type I receptors in brain cytosol. Neurochemical Research 13 707-713. (doi:10.1007/ BF00971592)

Fjeld CC, Birdsong WT \& Goodman RH 2003 Differential binding of $\mathrm{NAD}+$ and NADH allows the transcriptional corepressor carboxylterminal binding protein to serve as a metabolic sensor. PNAS 100 9202-9207. (Epub 2003 Jul 18) (doi:10.1073/pnas.1633591100)

Fuller PJ, Yang J \& Young MJ 201730 years of the mineralocorticoid receptor: coregulators as mediators of mineralocorticoid receptor signalling diversity. Journal of Endocrinology 234 T23-T35. (doi:10.1530/JOE-17-0060)

Published by Bioscientifica Ltd. 
Funder J \& Myles K 1996 Exclusion of corticosterone from epithelial mineralocorticoid receptors is insufficient for selectivity of aldosterone action: in vivo binding studies. Endocrinology 1137 5264-5268. (doi:10.1210/endo.137.12.8940344)

Funder JW, Feldman D \& Edelman IS 1972 Specific aldosterone binding in rat kidney and parotid. Journal of Steroid Biochemistry 3 209-218. (doi:10.1016/0022-4731(72)90052-0)

Funder JW, Pearce PT, Smith R \& Smith AI 1988 Mineralocorticoid action: target tissue specificity is enzyme, not receptor, mediated. Science $\mathbf{2 4 2}$ 583-585. (doi:10.1126/science.2845584)

Funder JW, Pearce PT, Smith R \& Campbell J 1989 Vascular type I aldosterone binding sites are physiological mineralocorticoid receptors. Endocrinology 125 2224-2226. (doi:10.1210/endo125-4-2224)

Ganong WF \& Mulrow PJ 1958 Rate of change in sodium and potassium excretion after injection of aldosterone into the aorta and renal artery of the dog. American Journal of Physiology 195 337-342.

Kagawa CM, Cella JA \& Van Arman CG 1957 Action of new steroids in blocking effects of aldosterone and desoxycorticosterone on salt. Science 126 1015-1016. (doi:10.1126/science.126.3281.1015)

Kassahn KS, Ragan MA \& Funder JW 2011 Mineralocorticoid receptors evolutionary and pathophysiological considerations. Endocrinology 152 1883-1890. (doi:10.1210/en.2010-1444, Epub 2011 Feb 22)

Krozowski Z \& Funder JW 1981 Mineralocorticoid receptors in rat anterior pituitary: toward a redefinition of 'mineralocorticoid hormone'. Endocrinology 109 1221-1224. (doi:10.1210/ endo-109-4-1221)
Mihailidou AS, Loan Le TY, Mardini M \& Funder JW 2009 Glucocorticoids activate cardiac mineralocorticoid receptors during experimental myocardial infarction. Hypertension 54 1306-1312. (doi:10.1161/ HYPERTENSIONAHA.109.136242, Epub 2009 Oct 19)

New MI \& Peterson RE 1967 A new form of congenital adrenal hyperplasia. Journal of Clinical Endocrinology and Metabolism 27 300-305. (doi:10.1210/jcem-27-2-300)

Pearce P \& Funder JW 1987 High affinity aldosterone binding sites (type I receptors) in rat heart. Clinical and Experimental Pharmacology and Physiology 14 859-866. (doi:10.1111/j.1440-1681.1987.tb02422.x)

Pitt B, Zannad F, Remme WJ, Cody R, Castaigne A, Perez A, Palensky J \& Wittes J 1999 The effect of spironolactone on morbidity and mortality in patients with severe heart failure. Randomized Aldactone Evaluation Study Investigators. New England Journal of Medicine 341 709-717. (doi:10.1056/NEJM199909023411001)

Rousseau G, Baxter JD, Funder JW, Edelman IS \& Tomkins GM 1972 Glucocorticoid and mineralocorticoid receptors for aldosterone. Journal of Steroid Biochemistry 3 219-227. (doi:10.1016/00224731(72)90053-2)

Shibata S, Rinehart J, Zhang J, Moeckel G, Castañeda-Bueno M, Stiegler AL, Boggon TJ, Gamba G \& Lifton RP 2013 Mineralocorticoid receptor phosphorylation regulates ligand binding and renal response to volume depletion and hyperkalemia. Cell Metabolism 18 660-671. (doi:10.1016/j.cmet.2013.10.005)

Sutherland DJ, Ruse JL \& Laidlaw JC 1966 Hypertension, increased aldosterone secretion and low plasma renin activity relieved by dexamethasone. Canadian Medical Association Journal 95 1109-1119.

Received in final form 09 May 2017

Accepted 19 May 2017

Accepted Preprint published online 22 May 2017
๑) 2017 Society for Endocrinology Printed in Great Britain 